\title{
Microstructural Evolution of High-Strain-Rate Severe Plastic Deformation Processed 316L during Kr Ion Irradiation and elevated Temperature Exposures
}

\author{
J.M.K. Wiezorek ${ }^{1}$, M.A, Gordillo ${ }^{1}$, M.A. Kirk ${ }^{2}$, P.M. Baldo ${ }^{3}$ \\ 1. Department of Mechanical Engineering and Materials Science, University of Pittsburgh, PA, USA \\ 2. Nuclear Engineering Division, Argonne National Laboratory, Lemont, IL, USA \\ 3. Materials Science Division, Argonne National Laboratory, Lemont, IL, USA
}

Austenitic stainless steels (SS) are used as structural materials in reactor internal components of nuclear power plants and also in fast reactors [1]. They degrade by prolonged elevated temperature exposure to neutron irradiation, leading to radiation enhanced kinetics for point defect migrations, manifest in hardening, embrittlement and void swelling, irradiation creep, radiation-induced segregation (RIS) and precipitation [1, 2]. RIS accelerated thermally-driven phase transformations are expected to become increasingly relevant for the degradation of austenitic SS under extended life conditions and development of materials with improved irradiation-damage tolerance is desirable [2]. Enhanced irradiation damage tolerance has been reported for materials with microstructures comprising high densities of sink sites for annihilation of excess point defects, e.g. intra-granularly provided by thermodynamically stable precipitates and inter-granularly as grain boundaries [3-7].

Here we report a transmission electron microscopy (TEM) based investigation of the microstructural development of 316L SS alloys modified by novel high-strain rate severe plastic deformation (SPD) methods based on linear plain strain processing under exposures to $\mathrm{Kr}$ ion-irradiation and elevated temperatures [8-11]. The SPD-modified SS offer significantly improved mechanical strength, fatigue performance, resist thermal coarsening up to $\sim 650^{\circ} \mathrm{C}$, and exhibit highly deformed, ultra-fine-grain to nano-scale refined grains of austenitic-Fe with lath-like morphology and trace amounts of martensite [8-11] (Fig. 1). In-situ irradiation TEM experiments were performed with $1 \mathrm{MeV} \mathrm{Kr}$ ions at $298 \mathrm{~K}, 573 \mathrm{~K}, 673 \mathrm{~K}$ and $773 \mathrm{~K}$ for fluence up to $\sim 1 \times 10^{17} \mathrm{ions} / \mathrm{cm}^{2}$, equivalent to about $20 \mathrm{dpa}$, using the IVEM-Tandem Facility at Argonne National Laboratory. In-situ sequences during heating and ion-irradiation have been recorded typically with a frame rate of 10 frames per second with $300 \mathrm{keV}$ electrons using a double-tilt heating holder. TEM analyses prior to and after the ion-irradiation exposures have been performed using a Jeol JEM2100F and a FEI Tecnai G2 F20ST operated at $200 \mathrm{kV}$. Experimental details for automated crystal orientation mapping (ACOM), which has been performed with NanoMegas ASTAR and TOPSPIN systems, and for TEM specimen preparation have been reported previously [8-11]. The 316L SS microstructure remained austenitic, resisted significant grain coarsening, retained its morphology and did not exhibit any precipitation of intermetallic or carbide phases after up to $\sim 6.5 \mathrm{~h}$ of $\mathrm{Kr}$ ion irradiation ( 20dpa) at up to $773 \mathrm{~K}$ (e.g. Fig. 2). In-situ TEM revealed the formation of interstitial dislocation loops and interactions of the irradiation induced defects with each other and the pre-existing intra-granular dislocation structures and the grain boundaries, leading to reduction in dislocation density and local grain disorientations. The dynamic in-situ TEM observations enabled identification of different fluence dependent regimes during irradiation damage evolution for the different temperatures ranging from $298 \mathrm{~K}$ to $773 \mathrm{~K}$. Complementing TEM based analyses prior to and after ion irradiation enabled quantitative measurements of grain size, locally nano-meter scale resolved grain orientations and grain boundary network structure. The microstructure evolution of the modified 316L during irradiation has been 
studied with a specific focus on the role of changes to the grain boundary structure and the intragranular dislocation populations. [12]

References:

[1] E.A. Kenik, J.T. Busby, Mat. Sci. Eng. R 73 (2012), p. 67

[2] X. Zhang et al. JOM 62 (2010), p. 75

[3] C. Sun et al. J. Nucl. Mat. 420 (2012), p. 235

[4] P.B. Revathy Rajan et al. IOP Conference Series: Mat. Sci. Eng 63 (2014), p. 012121

[5] B. Singh, A. Foreman Phil. Mag. 29 (1974), p. 847

[6] M. Song et al. Acta Materialia 74 (2014), p. 285

[7] C. Sun et al. Sci. Rep. 5 (2015), 7801; DOI:10.1038/srep07801

[8] Y. Idell et al. Scri. Mat. 68 (2013), p. 667

[9] Y. Idell, 'Thermo-Mechanical Processing of Austenitic Steel to Mitigate Surface Related

Degradation', PhD Thesis (2014), University of Pittsburgh

[10] Y. Idell, J.M.K. Wiezorek, Microsc. Microanal. 20 S3 (2014), p. 1446

[11] C. Ye et al. Mat. Sci. Eng. A 613 (2014), p. 274

[12] Support for the research from Nuclear Regulatory Commission and access to the electron microscopy with ion irradiation offered by the IVEM-Tandem Facility at Argonne National Laboratory, a U.S. Department of Energy Facility funded by the DOE Office of Nuclear Energy, operated under Contract No. DE-AC02-06CH11357 by UChicago Argonne, LLC, as well as the facilities of the Materials Micro-Characterization Laboratory of the Department of Mechanical Engineering and Materials Science at the University of Pittsburgh are gratefully acknowledged.
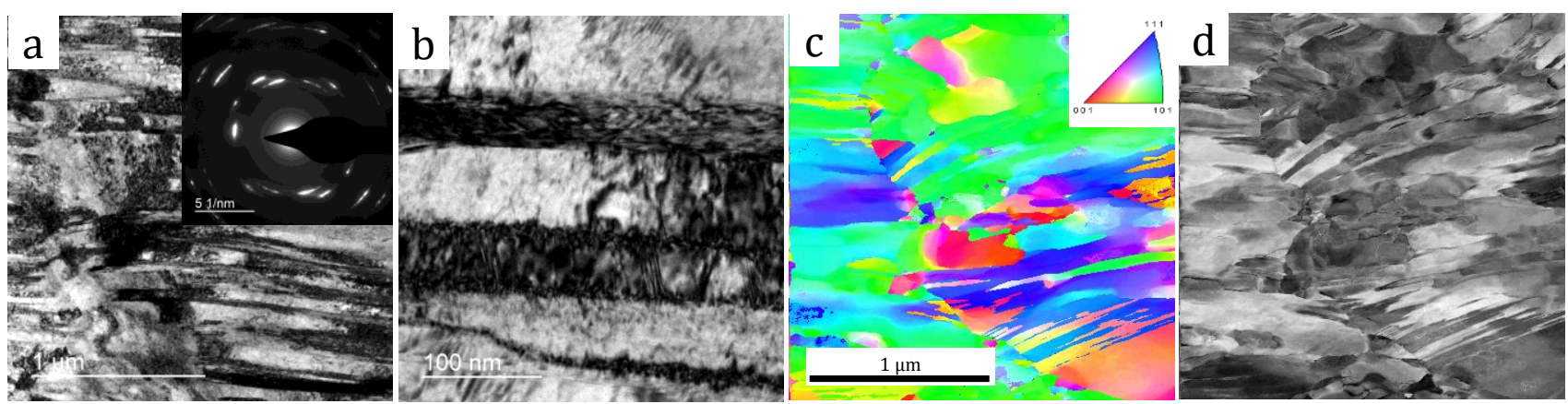

Figure 1. Bright field TEM (a), (b), precession electron diffraction based (ACOM) orientation map (c), and indexing reliability map (d), for the modified 316L prior to irradiations.
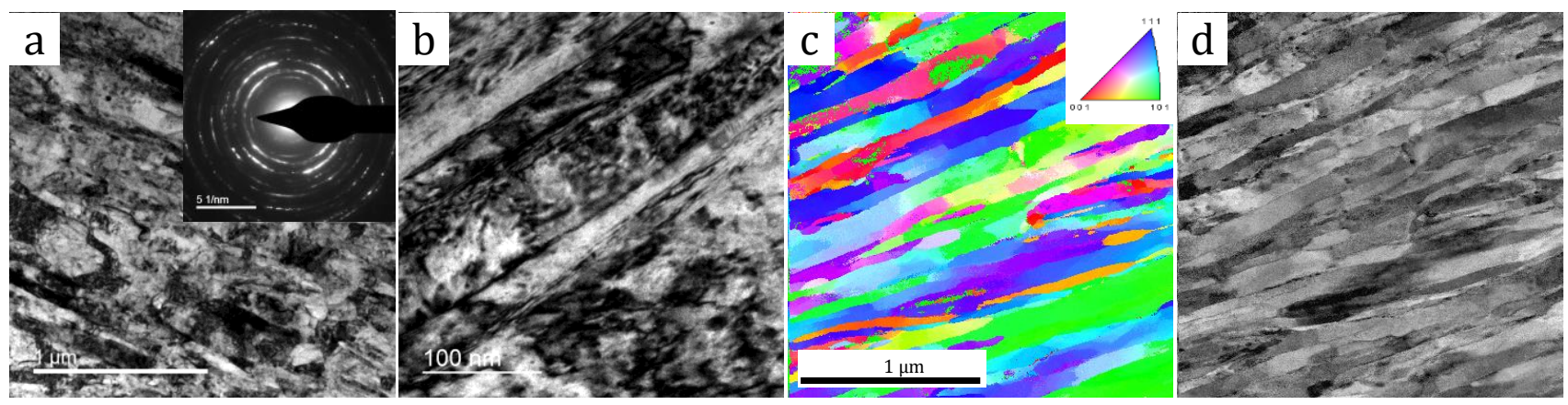

Figure 2. Bright field TEM (a), (b), precession electron diffraction based (ACOM) orientation map (c), and indexing reliability map (d), for the modified $316 \mathrm{~L}$ after $573 \mathrm{~K} \mathrm{Kr}$-ion irradiations to $20 \mathrm{dpa}$. 